Research Article

\title{
On the Generalized KdV Hierarchy and Boussinesq Hierarchy with Lax Triple
}

\author{
Xiaoli Wang ${ }^{1, *},\left(\mathbb{D}\right.$, , Jian-Qin $\mathrm{Mei}^{2}$ \\ ${ }^{1}$ School of Mathematics and Statistics, Qilu University of Technology (Shandong Academy of Sciences), Jinan 250353, China \\ ${ }^{2}$ School of Mathematical Sciences, Dalian University of Technology, Dalian 116024, China
}

ARTICLE INFO
Article History
Received 11 March 2021
Accepted 31 May 2021
Keywords
Lax equation
KdV hierarchy
Boussinesq hierarchy
Lax triple
2000 Mathematics Subject
Classifications
37K10
35Q53

\section{ARTICLE INFO}

$35 \mathrm{Q} 53$

\section{ABSTRACT}

Based on the Nambu 3-bracket and the operators of the KP hierarchy, we propose the generalized Lax equation of the Lax triple. Under the operator constraints, we construct the generalized KdV hierarchy and Boussinesq hierarchy. Moreover, we present the exact solutions of some nonlinear evolution equations.

(C) 2021 The Authors. Published by Atlantis Press B.V. This is an open access article distributed under the CC BY-NC 4.0 license (http://creativecommons.org/licenses/by-nc/4.0/).

\section{INTRODUCTION}

Nambu mechanics [8] is a generalization of classical Hamiltonian mechanics based on Liouville theorem. Poisson brackets in Hamilton mechanics are replaced by Nambu brackets. Based on Nambu brackets, Nambu 3-algebra [10] is introduced. It is a natural generalization of Lie Algebra with high structure. 3-algebra has been widely applied in string theory and M-branches [1,9]. In recent years, the relationship between infinite dimensional 3-algebra and integrable system has attracted wide attention in the framework of Nambu mechanics $[2,3,14]$.

The Kadomtsev-Petviashvili (KP) hierarchy [5-7,13] is an important classical integrable system. There are different approaches to the description of the KP hierarchy. One of them is described in terms of a Lax pair $\left(B_{n}, L\right)$. By means of the operator Nambu 3-bracket, the generalized Lax equation of the KP hierarchy with the Lax triple $\left(B_{m}, B_{n}, L\right)$ was studied in [12], where the KP equation and other integrable (nonintegrable) equations were derived, and the soliton wave solutions of the nonlinear evolution equations were provided. The BKP and CKP hierarchies are two important reductions of the KP hierarchy. When the operator $L$ satisfies the constraints $L^{*}=-\partial L \partial^{-1}$ and $L^{*}=-L$, the KP hierarchy becomes the BKP and CKP hierarchies, respectively. The dKP hierarchy is the quasi classical limit of the KP hierarchy. Based on the Lax triple $\left(B_{m}, B_{n}, L\right)$, the generalized BKP, CKP and dKP hierarchies were investigated [4,11]. When the operator $L$ satisfies the constraints $\left(L^{2}\right)_{-}=0$ and $\left(L^{3}\right)_{-}=0$, the KP hierarchy becomes the KdV and Boussinesq hierarchies, respectively. Both KdV equation and Boussinesq equation are derived from the study of shallow water waves. They both contain N-soliton solutions. Boussinesq equation can be considered as a generalization of $\mathrm{KdV}$ equation, which allows solitons to propagate in two directions. The aim of this paper is to derive the nonlinear evolution equations from the generalized Lax equation in term of the Lax triple $\left(B_{m}, B_{n}, L\right)$ of the KdV and Boussinesq hierarchies.

This paper is arranged as follows. In Section 2, the generalized Lax equation of KP hierarchy and operator constraints is introduced. In Sections 3 and 4, we give the generalized KdV and Boussinesq hierarchies, respectively. Finally, a short conclusion and further discussion are presented. 


\section{GENERALIZED LAX EQUATION}

The KP hierarchy can be derived from the well-known Lax equation,

$$
\frac{\partial L}{\partial t_{n}}=\left[B_{n}, L\right]=B_{n} L-L B_{n}, \quad n=1,2, \cdots .
$$

Here $B_{n}=\left(L^{n}\right)_{+}, \quad n \geq 1 . L$ is a pseudo-differential operator,

$$
L=\partial+\sum_{i=0}^{+\infty} v_{i}(t) \partial^{-i-1},
$$

where $t=\left(t_{1}, t_{2}, \cdots\right)$ are the time variables and $\partial=\partial / \partial_{x}, x=t_{1}$, the negative powers of $\partial$ are to be understood as the formal integration symbols.

As the operator $L$ satisfies the constraints $\left(L^{2}\right)_{-}=0$ and $\left(L^{3}\right)_{-}=0$, respectively, we can derive the KdV hierarchy and Boussinesq hierarchy from the Lax equation (2.1). Here $\left(L^{k}\right)_{-}, k=2,3$, denotes the integral part of $L^{k}$,

$$
\left(L^{k}\right)_{-}=L^{k}-\left(L^{k}\right)_{+}=L^{k}-B_{k}
$$

The constraints $\left(L^{k}\right)_{-}=0$ means $\left[B_{k n}, B_{k}\right]=0, n=1,2, \ldots$, thus we can derive

$$
\frac{\partial L^{k}}{\partial t_{k n}}=0, k=2,3, n=1,2, \ldots
$$

Based on the operator Nambu 3-bracket, the generalized Lax equation with respect to the Lax triple $\left(L, B_{n}, B_{m}\right)[12]$ is defined by

$$
\frac{\partial L}{\partial t_{m n}}=\left[B_{m}, B_{n}, L\right]_{-}, \quad(m, n=0,1,2 \cdots),
$$

where $B_{0}=1$. The operator Nambu 3-bracket [, , ]_ denotes the formal integration operator part of the derived pseudo-differential operator.

Taking $B_{m}=B_{0}$ in (2.4), it is easy to verify that (2.4) leads to the Lax equation (2.1). Thus it is natural to derive the KP hierarchy from (2.4). As the operator $L$ satisfy the constraints $\left(L^{2}\right)_{-}=0$ and $\left(L^{3}\right)_{-}=0$, respectively, we can also derive the KdV hierarchy and Boussinesq hierarchy.

In the following, we will list the usual KdV hierarchy and Boussinesq hierarchy. And we also will derive the generalized KdV hierarchy and Boussinesq hierarchy from the generalized Lax equation (2.4).

\section{GENERALIZED KdV HIERARCHY}

Equating the coefficients of the operator $\partial^{-i}(i=1,2, \cdots)$ in the constraints $\left(L^{2}\right)_{-}=0$, we can derive

$$
\begin{aligned}
& v_{1}=-\frac{1}{2} v_{0, x}, \\
& v_{2}=-\frac{1}{2} v_{0}^{2}+\frac{1}{4} v_{0, x x}, \\
& v_{3}=\frac{3}{2} v_{0} v_{0, x}-\frac{1}{8} v_{0, x x x}, \\
& v_{4}=-\frac{7}{4} v_{0} v_{0, x x}+\frac{1}{2} v_{0}^{3}-\frac{11}{8} v_{0, x}^{2}+\frac{1}{16} v_{0, x x x x}, \\
& v_{5}=\frac{15}{8} v_{0} v_{0, x x x}+\frac{15}{4} v_{0, x} v_{0, x x}-\frac{15}{4} v_{0, x} v_{0}^{2}-\frac{1}{32} v_{0, x x x x x},
\end{aligned}
$$

Then we can derive $B_{n}$ of the KdV hierarchy are

$$
\begin{aligned}
& B_{1}=\partial, \\
& B_{2}=\partial^{2}+2 v_{0} \\
& B_{3}=\partial^{3}+3 v_{0} \partial+\frac{3}{2} v_{0, x}, \\
& B_{4}=\partial^{4}+4 v_{0} \partial^{2}+4 v_{0, x} \partial+4 v_{0}^{2}+2 v_{0, x x} \\
& B_{5}=\partial^{5}+5 v_{0} \partial^{3}+\frac{15}{2} v_{0, x} \partial^{2}+\left(\frac{15}{2} v_{0}^{2}+\frac{25}{4} v_{0, x x}\right) \partial+\frac{15}{2} v_{0} v_{0, x}+\frac{15}{8} v_{0, x x x},
\end{aligned}
$$




$$
\begin{aligned}
B_{6}= & \partial^{6}+6 v_{0} \partial^{4}+12 v_{0, x} \partial^{3}+\left(12 v_{0}^{2}+14 v_{0, x x}\right) \partial^{2}+\left(24 v_{0} v_{0, x}+8 v_{0, x x x}\right) \partial \\
& +2 v_{0, x x x x}+12 v_{0} v_{0, x x}+8 v_{0}^{3}+8 v_{0, x}^{2} \\
B_{7}= & \partial^{7}+7 v_{0} \partial^{5}+\frac{35}{2} v_{0, x} \partial^{4}+\left(\frac{35}{2} v_{0}^{2}+\frac{105}{4} v_{0, x x}\right) \partial^{3}+\left(\frac{105}{2} v_{0} v_{0, x}+\frac{175}{8} v_{0, x x x}\right) \partial^{2} \\
& +\left(\frac{35}{2} v_{0}^{3}+\frac{175}{4} v_{0} v_{0, x x}+\frac{245}{8} v_{0, x}^{2}+\frac{161}{16} v_{0, x x x x}\right) \partial+\frac{63}{32} v_{0, x x x x x} \\
& +\frac{105}{8} v_{0} v_{0, x x x}+\frac{105}{4} v_{0, x} v_{0, x x}+\frac{105}{4} v_{0}^{2} v_{0, x} \\
& \vdots
\end{aligned}
$$

Taking $B_{m}=B_{0}$, we list some evolution equations of the KdV hierarchy as follows:

- For the case of $B_{n}=B_{3}$, we have

$$
\frac{\partial v_{0}}{\partial t_{03}}=\frac{1}{4} v_{0, x x x}+3 v_{0, x} v_{0}
$$

which is the well-known $\mathrm{KdV}$ equation. Under the scaling transformation $v_{0}=\frac{1}{2} u, t_{03}=4 t$, (3.3) becomes the usual KdV equation.

- For the case of $B_{n}=B_{5}$, we have

$$
\frac{\partial v_{0}}{\partial t_{05}}=\frac{1}{16} v_{0, x x x x x}+\frac{5}{4} v_{0} v_{0, x x x}+\frac{5}{2} v_{0, x} v_{0, x x}+\frac{15}{2} v_{0, x} v_{0}^{2} .
$$

Under the scaling transformation $v_{0}=\frac{1}{2} u, t_{05}=16 t$, (3.4) becomes the usual 5-order KdV equation.

- For the case of $B_{n}=B_{7}$, we have

$$
\begin{aligned}
\frac{\partial v_{0}}{\partial t_{07}}= & \frac{1}{64} v_{0, x x x x x x x}+\frac{7}{16} v_{0} v_{0, x x x x x}+\frac{21}{16} v_{0, x} v_{0, x x x x}+\frac{35}{16} v_{0, x x} v_{0, x x x} \\
& +\frac{35}{2} v_{0} v_{0, x} v_{0, x x}+\frac{35}{8} v_{0}^{2} v_{0, x x x}+\frac{35}{8} v_{0, x}^{3}+\frac{35}{2} v_{0, x} v_{0}^{3} .
\end{aligned}
$$

Under the scaling transformation $v_{0}=\frac{1}{2} u, t_{07}=64 t$, (3.5) becomes the usual 7-order KdV equation.

In the following, we will list some evolution equations of the generalized KdV hierarchy from the generalized Lax equation (2.4) except $B_{m}=B_{0}$. We also get the single soliton solution of some nonlinear evolution equations.

- Taking the operator pair $\left(B_{1}, B_{2}\right)$ in (2.4), we have

$$
\frac{\partial v_{0}}{\partial t_{12}}=-\frac{1}{4} v_{0, x x x}+v_{0} v_{0, x}
$$

Under the scaling transformation $v_{0}=-\frac{3}{2} u, t_{12}=-4 t$, (3.6) becomes the usual KdV equation.

- Taking the operator pair $\left(B_{1}, B_{3}\right)$ in (2.4), we have

$$
\frac{\partial v_{0}}{\partial t_{13}}=0
$$

- Taking the operator pair $\left(B_{1}, B_{4}\right)$ in (2.4), we have

$$
\frac{\partial v_{0}}{\partial t_{14}}=-\frac{1}{16} v_{0, x x x x x}+\frac{11}{4} v_{0} v_{0, x x x}+\frac{7}{2} v_{0, x} v_{0, x x}+\frac{9}{2} v_{0, x} v_{0}^{2} .
$$

Its single soliton solution is

$$
v_{0}=\frac{5\left(3 \operatorname{sech}^{2} \xi-1\right)(5 \sqrt{41}-33) k^{2}}{-21+9 \sqrt{41}}
$$

where $\xi=k(\omega t+x)+b$ in which

$$
\omega=\frac{(-1019699+159231 \sqrt{41}) k^{4}}{-33606+5214 \sqrt{41}}
$$

$b$ and $k$ are arbitrary constants.

- Taking the operator pair $\left(B_{2}, B_{3}\right)$ in (2.4), we have

$$
\frac{\partial v_{0}}{\partial t_{23}}=\frac{1}{16} v_{0, x x x x x}+\frac{1}{2} v_{0} v_{0, x x x}+\frac{9}{4} v_{0, x} v_{0, x x}-3 v_{0, x} v_{0}^{2} .
$$


Its single soliton solution is

$$
v_{0}=-\frac{5 k^{2}}{2}\left(3 \sec h^{2} \xi-1\right),
$$

where $\xi=k(\omega t+x)+b$ in which $\omega=-\frac{51 k^{4}}{4}, b$ and $k$ are arbitrary constants.

- Taking the operator pairs $\left(B_{1}, B_{5}\right)$ and $\left(B_{2}, B_{4}\right)$ in $(2.4)$, we have

- Taking the operator pair $\left(B_{1}, B_{6}\right)$ in (2.4), we have

$$
\frac{\partial v_{0}}{\partial t_{15}}=\frac{\partial v_{0}}{\partial t_{24}}=0 .
$$

$$
\begin{aligned}
\frac{\partial v_{0}}{\partial t_{16}}= & -\frac{1}{64} v_{0, x x x x x x x}+\frac{57}{16} v_{0} v_{0, x x x x x}+\frac{119}{16} v_{0, x} v_{0, x x x x}+\frac{125}{16} v_{0, x x} v_{0, x x x} \\
& +\frac{35}{2} v_{0} v_{0, x} v_{0, x x}+\frac{45}{8} v_{0}^{2} v_{0, x x x}+\frac{25}{8} v_{0, x}^{3}+\frac{25}{2} v_{0, x} v_{0}^{3} .
\end{aligned}
$$

- Taking the operator pair $\left(B_{2}, B_{5}\right)$ in $(2.4)$, we have

$$
\begin{aligned}
\frac{\partial v_{0}}{\partial t_{25}}= & \frac{1}{64} v_{0, x x x x x x x}+\frac{1}{4} v_{0} v_{0, x x x x x}+\frac{5}{4} v_{0, x} v_{0, x x x x}+\frac{35}{16} v_{0, x x} v_{0, x x x} \\
& +\frac{35}{4} v_{0} v_{0, x} v_{0, x x}+\frac{5}{8} v_{0}^{2} v_{0, x x x}+\frac{15}{4} v_{0, x}^{3}-\frac{15}{2} v_{0, x} v_{0}^{3} .
\end{aligned}
$$

- Taking the operator pair $\left(B_{3}, B_{4}\right)$ in $(2.4)$, we have

$$
\begin{aligned}
\frac{\partial v_{0}}{\partial t_{34}}= & -\frac{1}{64} v_{0, x x x x x x x}+\frac{1}{2} v_{0} v_{0, x x x x x}-\frac{1}{4} v_{0, x} v_{0, x x x x}-\frac{27}{16} v_{0, x x} v_{0, x x x} \\
& +\frac{141}{4} v_{0} v_{0, x} v_{0, x x}+\frac{75}{8} v_{0}^{2} v_{0, x x x}+\frac{33}{4} v_{0, x}^{3}+\frac{27}{2} v_{0, x} v_{0}^{3} .
\end{aligned}
$$

- Taking the operator pairs $\left(B_{1}, B_{7}\right),\left(B_{2}, B_{6}\right)$ and $\left(B_{3}, B_{5}\right)$ in $(2.4)$, we have

$$
\frac{\partial v_{0}}{\partial t_{17}}=\frac{\partial v_{0}}{\partial t_{26}}=\frac{\partial v_{0}}{\partial t_{35}}=0 .
$$

From the above evolution equations, we can conjecture that when $m+n$ is even, the nonlinear evolution equation is $\frac{\partial v_{0}}{\partial t_{m n}}=0$.

\section{GENERALIZED BOUSSINESQ HIERARCHY}

Equating the coefficients of the operator $\partial^{-i}(i=1,2, \cdots)$ in the constraints $\left(L^{3}\right)_{-}=0$, we can derive

$$
\begin{aligned}
v_{2}= & -v_{0}^{2}-\frac{1}{3} v_{0, x x}-v_{1, x}, \\
v_{3}= & 2 v_{0} v_{0, x}+\frac{1}{3} v_{0, x x x}-2 v_{0} v_{1}+\frac{2}{3} v_{1, x x} \\
v_{4}= & -v_{0} v_{0, x x}+\frac{5}{3} v_{0}^{3}-v_{0, x}^{2}-\frac{2}{9} v_{0, x x x x}+4 v_{0} v_{1, x}+3 v_{1} v_{0, x}-v_{1}^{2}-\frac{1}{3} v_{1, x x x}, \\
v_{5}= & -10 v_{0, x} v_{0}^{2}+5 v_{0}^{2} v_{1}+5 v_{1} v_{1, x}-\frac{20}{3} v_{0, x} v_{1, x}-\frac{10}{3} v_{1} v_{0, x x}-5 v_{0} v_{1, x x} \\
& +\frac{1}{9} v_{0, x x x x x}+\frac{1}{9} v_{1, x x x x}, \\
& \vdots
\end{aligned}
$$

Then we can derive $B_{n}$ of the Boussinesq hierarchy are

$$
\begin{aligned}
B_{1}= & \partial \\
B_{2}= & \partial^{2}+2 v_{0}, \\
B_{3}= & \partial^{3}+3 v_{0} \partial+3 v_{0, x}+3 v_{1}, \\
B_{4}= & \partial^{4}+4 v_{0} \partial^{2}+\left(4 v_{1}+6 v_{0, x}\right) \partial+2 v_{0}^{2}+\frac{8}{3} v_{0, x x}+2 v_{1, x}, \\
B_{5}= & \partial^{5}+5 v_{0} \partial^{3}+\left(5 v_{1}+10 v_{0, x}\right) \partial^{2}+\left(5 v_{0}^{2}+\frac{25}{3} v_{0, x x}+5 v_{1, x}\right) \partial+10 v_{0} v_{1} \\
& +\frac{10}{3} v_{1, x x}+10 v_{0} v_{0, x}+\frac{10}{3} v_{0, x x x},
\end{aligned}
$$


Similarly, when $m=0$, we can list some evolution equations of the Boussinesq hierarchy as follows:

- For the case of $B_{n}=B_{2}$, we have

$$
\begin{aligned}
& \frac{\partial v_{0}}{\partial t_{02}}=v_{0, x x}+2 v_{1, x} \\
& \frac{\partial v_{1}}{\partial t_{02}}=-\frac{2}{3} v_{0, x x x}-2 v_{0, x} v_{0}-v_{1, x x},
\end{aligned}
$$

Eliminating $v_{1}$, replacing $v_{0}$ with $-u$, and replacing $t_{02}$ with $t$, we can get

$$
3 \frac{\partial^{2} u}{\partial t^{2}}+\left(u_{x x x}-12 u u_{x}\right)_{x}=0
$$

which is the well-known Boussinesq equation.

- For the case of $B_{n}=B_{4}$, we have

$$
\begin{aligned}
& \frac{\partial v_{0}}{\partial t_{04}}=\left(\frac{1}{3} v_{0, x x x}+2 v_{0} v_{0, x}+4 v_{0} v_{1}+\frac{2}{3} v_{1, x x}\right)_{x}, \\
& \frac{\partial v_{1}}{\partial t_{04}}=-\frac{2}{9} v_{0, x x x x x}-2 v_{0} v_{0, x x x}-4_{0, x} v_{0, x x}-4 v_{0}^{2} v_{0, x}-2\left(v_{0} v_{1, x}\right)_{x}-\frac{1}{3} v_{1, x x x x}+4 v_{1} v_{1, x} .
\end{aligned}
$$

Under the scaling transformation $v_{0}=\frac{1}{3} u, v_{1}=-\frac{1}{3} v, t_{04}=-t,(4.5)$ becomes the second equation of the Boussinesq hierarchy,

$$
\begin{aligned}
& \frac{\partial u}{\partial t}=\left(-\frac{1}{3} u_{x x x}-\frac{2}{3} u u_{x}+\frac{4}{3} u v+\frac{2}{3} v_{x x}\right)_{x}, \\
& \frac{\partial v}{\partial t}=-\frac{2}{9} u_{x x x x x}-\frac{2}{3} u u_{x x x}-\frac{4}{3} u_{x} u_{x x}-\frac{4}{9} u^{2} u_{x}+\frac{2}{3}\left(u v_{x}\right)_{x}+\frac{1}{3} v_{x x x x}+\frac{4}{3} v v_{x} .
\end{aligned}
$$

- For the case of $B_{n}=B_{5}$, we have

$$
\begin{aligned}
& \frac{\partial v_{0}}{\partial t_{05}}=10 v_{1} v_{1, x}-5 v_{0, x} v_{0}^{2}+5\left(v_{0, x} v_{1}\right)_{x}-\frac{5}{3}\left(v_{0} v_{0, x x}\right)_{x}-\frac{1}{9} v_{0, x x x x x}, \\
& \frac{\partial v_{1}}{\partial t_{05}}=\left(-5 v_{1} v_{1, x}-5 v_{0}^{2} v_{1}-\frac{10}{3}\left(v_{0, x} v_{1}\right)_{x}-\frac{5}{3} v_{0} v_{1, x x}-\frac{1}{9} v_{1, x x x x}\right)_{x} .
\end{aligned}
$$

Under the scaling transformation $v_{0}=\frac{1}{3} u, v_{1}=-\frac{1}{3} v, t_{05}=-t,(4.7)$ becomes the third equation of the Boussinesq hierarchy,

$$
\begin{aligned}
& \frac{\partial u}{\partial t}=-\frac{10}{3} v v_{x}+\frac{5}{9} u_{x} u^{2}+\frac{5}{3}\left(u_{x} v\right)_{x}+\frac{5}{9}\left(u u_{x x}\right)_{x}+\frac{1}{9} u_{x x x x x}, \\
& \frac{\partial v}{\partial t}=\left(-\frac{5}{3} v v_{x}+\frac{5}{9} u^{2} v+\frac{10}{9}\left(u_{x} v\right)_{x}+\frac{5}{9} u v_{x x}+\frac{1}{9} v_{x x x x}\right)_{x} .
\end{aligned}
$$

In the following, we will list some evolution equations of the generalized Boussinesq hierarchy from the generalized Lax equation (2.4) except $B_{m}=B_{0}$.

- Taking the operator pair $\left(B_{1}, B_{2}\right)$ in $(2.4)$, we have

$$
\begin{aligned}
& \frac{\partial v_{0}}{\partial t_{12}}=-\frac{1}{3} v_{0, x x x}, \\
& \frac{\partial v_{1}}{\partial t_{12}}=4 v_{1} v_{0, x}-4 v_{0} v_{1, x}-2 v_{0} v_{0, x x}+2 v_{0, x}^{2}-\frac{1}{3} v_{1, x x x} .
\end{aligned}
$$

- Taking the operator pair $\left(B_{1}, B_{3}\right)$ in $(2.4)$, we have

$$
\begin{aligned}
& \frac{\partial v_{0}}{\partial t_{13}}=\left(2 v_{0} v_{1}+v_{0} v_{0, x}-\frac{2}{3} v_{1, x x}-\frac{1}{3} v_{0, x x x}\right)_{x}, \\
& \frac{\partial v_{1}}{\partial t_{13}}=\left(2 v_{0} v_{0, x x}+\frac{2}{9} v_{0, x x x x}+\frac{1}{3} v_{1, x x x}-v_{0} v_{1, x}+v_{1}^{2}-\frac{2}{3} v_{0}^{3}\right)_{x} .
\end{aligned}
$$

- Taking the operator pair $\left(B_{1}, B_{4}\right)$ in (2.4), we have

$$
\begin{aligned}
\frac{\partial v_{0}}{\partial t_{14}}= & \left(-v_{0}^{3}+3 v_{1}^{2}+3 v_{1} v_{0, x}+3 v_{0} v_{0, x x}+2 v_{0, x}^{2}\right)_{x}, \\
\frac{\partial v_{1}}{\partial t_{14}}= & -3\left(v_{1} v_{1, x}\right)_{x}-6 v_{1} v_{0} v_{0, x}+6 v_{1} v_{0, x x x}-4 v_{0, x x} v_{0}^{2}-11 v_{1, x} v_{0}^{2}+\frac{7}{3} v_{1, x x} v_{0, x} \\
& +4 v_{0, x x} v_{1, x}+\frac{5}{3} v_{0} v_{1, x x x}+\frac{2}{3} v_{0, x} v_{0, x x x}-\frac{2}{3} v_{0} v_{0, x x x x} .
\end{aligned}
$$


- Taking the operator pair $\left(B_{2}, B_{3}\right)$ in (2.4), we have

$$
\begin{aligned}
\frac{\partial v_{0}}{\partial t_{23}}= & \left(-\frac{4}{3} v_{0}^{3}+2 v_{1}^{2}+2 v_{1} v_{0, x}+v_{0} v_{0, x x}+2 v_{0, x}^{2}+\frac{1}{9} v_{0, x x x x}\right)_{x}, \\
\frac{\partial v_{1}}{\partial t_{23}}= & -2 v_{1, x}^{2}-5 v_{1} v_{1, x x}+2 v_{1} v_{0} v_{0, x}-\frac{7}{3} v_{1} v_{0, x x x}+3 v_{0, x x} v_{0}^{2}+2 v_{1, x} v_{0}^{2}+4 v_{1, x x} v_{0, x} \\
& +v_{0, x x} v_{1, x}+5 v_{0} v_{1, x x x}-\frac{2}{3} v_{0, x} v_{0, x x x}+2 v_{0} v_{0, x x x x}+2 v_{0, x}^{2} v_{0}-v_{0, x x}^{2}+\frac{1}{9} v_{1, x x x x x} .
\end{aligned}
$$

- Taking the operator pair $\left(B_{1}, B_{5}\right)$ in $(2.4)$, we have

$$
\begin{aligned}
\frac{\partial v_{0}}{\partial t_{15}}= & -\frac{1}{9} v_{0, x x x x x x}+\frac{20}{3} v_{0, x x}^{2}-\frac{2}{9} v_{1, x x x x x}+\frac{20}{3} v_{0, x} v_{1, x x}+\frac{40}{3} v_{0, x x} v_{1, x} \\
& +\frac{10}{3} v_{0} v_{1, x x x}+10 v_{1} v_{0, x x x}+\frac{25}{3} v_{0, x x x} v_{0, x}+\frac{5}{3} v_{0} v_{0, x x x x}, \\
\frac{\partial v_{1}}{\partial t_{15}}= & \frac{1}{9} v_{1, x x x x x x}+\frac{2}{27} v_{0, x x x x x x x}-\frac{100}{3} v_{0} v_{0, x} v_{0, x x}-20 v_{1, x} v_{1} v_{0}-10 v_{1, x} v_{0, x} v_{0} \\
& +\frac{10}{9} v_{0, x x x x} v_{0, x}-\frac{70}{9} v_{0, x x x} v_{0, x x}+\frac{4}{3} v_{0} v_{0, x x x x x}-10 v_{0}^{2} v_{0, x x x}+\frac{20}{3} v_{1, x} v_{1, x x} \\
& +\frac{20}{3} v_{0}^{3} v_{0, x}+10 v_{1}^{2} v_{0, x}+10 v_{1} v_{0, x}^{2}-\frac{25}{3} v_{0, x x x} v_{1, x}+10 v_{1} v_{1, x x x} \\
& -\frac{5}{3} v_{0} v_{1, x x x x}-\frac{20}{3} v_{1, x x} v_{0, x x}-\frac{10}{3} v_{0, x}^{3}-10 v_{0, x x} v_{1} v_{0} .
\end{aligned}
$$

- Taking the operator pair $\left(B_{2}, B_{4}\right)$ in (2.4), we have

$$
\begin{aligned}
\frac{\partial v_{0}}{\partial t_{24}}= & \frac{1}{9} v_{0, x x x x x x}+3 v_{0, x x}^{2}+\frac{2}{9} v_{1, x x x x x}+13 v_{0, x} v_{1, x x}+6 v_{0, x x} v_{1, x}+\frac{22}{3} v_{0} v_{1, x x x}+\frac{1}{3} v_{1} v_{0, x x x} \\
& +\frac{20}{3} v_{0, x x x} v_{0, x}+\frac{11}{3} v_{0} v_{0, x x x x}-12 v_{1} v_{0, x} v_{0}-3 v_{0, x x} v_{0}^{2}-6 v_{1, x} v_{0}^{2}-6 v_{0, x}^{2} v_{0} \\
\frac{\partial v_{1}}{\partial t_{24}}= & -\frac{1}{9} v_{1, x x x x x x}-\frac{2}{27} v_{0, x x x x x x x}-22 v_{0} v_{0, x} v_{0, x x}-12 v_{1, x} v_{1} v_{0}+6 v_{1, x} v_{0, x} v_{0} \\
& -\frac{10}{3} v_{0, x x x x} v_{0, x}-\frac{8}{3} v_{0, x x x} v_{0, x x}-\frac{8}{3} v_{0} v_{0, x x x x x}-\frac{16}{3} v_{0}^{2} v_{0, x x x}+\frac{71}{3} v_{1, x} v_{1, x x} \\
& +6 v_{0}^{3} v_{0, x}-16 v_{1}^{2} v_{0, x}-10 v_{1} v_{0, x}^{2}+\frac{26}{3} v_{0, x x x} v_{1, x}+\frac{41}{3} v_{1} v_{1, x x x}-\frac{11}{3} v_{0} v_{1, x x x x} \\
& +\frac{7}{3} v_{1, x x} v_{0, x x}-\frac{20}{3} v_{0, x}^{3}+3 v_{1, x x} v_{0}^{2}-\frac{10}{3} v_{1, x x x} v_{0, x}+\frac{20}{3} v_{1} v_{0, x x x x}
\end{aligned}
$$

\section{SUMMARY}

In this paper, in terms of the Lax triple $\left(B_{m}, B_{n}, L\right)$, we investigated the generalized Lax equation of the KdV and Boussinesq hierarchies. When $m=0$, the generalized Lax equation reduces to the usual Lax equation. We derived integrable evolution equations from the KdV and Boussinesq hierarchies. We got some soliton wave solutions from the nonlinear evolution equations of the generalized KdV hierarchy. Moreover, the evolution equations for the generalized KdV hierarchy seemed to be $\frac{\partial v_{0}}{\partial t_{m n}}=0$ when $m+n$ is even. We also derived some generalized nonlinear evolution equations from the generalized Boussinesq Lax equation. More properties of the generalized KdV and Boussinesq hierarchies still deserve further study.

\section{CONFLICTS OF INTEREST}

The authors declare they have no conflicts interest.

\section{AUTHORS' CONTRIBUTION}

All authors completed the paper together. All authors read and approved the final manuscript. 


\section{FUNDING}

This work is partially supported by National Natural Science Foundation of China (Grant No. 11801292) and the Fundamental Research Funds for the Central Universities (DUT19LK26).

\section{ACKNOWLEDGMENTS}

We thank the valuable suggestions of the referees.

\section{REFERENCES}

[1] C.H. Chen, K. Furuuchi, P.M. Ho, T. Takimi, More on the Nambu-Poisson M5-brane theory: scaling limit, background independence and an all order solution to the Seiberg-Witten map, J. High Energ. Phys. 10 (2010), 100.

[2] M.R. Chen, S.K. Wang, K. Wu, W.Z. Zhao, Infinite-dimensional 3-algebra and integrable system, J. High Energ. Phys. 12 (2012), 30.

[3] M.R. Chen, S.K. Wang, X.L. Wang, K. Wu, W.Z. Zhao, On $W_{1+\infty}$ 3-algebra and integrable system, Nucl. Phys. B 891 (2015), $655-675$.

[4] M.R. Chen, Y. Chen, Z.W. Yan, J.Q. Mei, X.L. Wang, A 3-Lie algebra and the dKP hierarchy, J. Nonlinear Math. Phys. 26 (2019), 91-97.

[5] E. Date, M. Kashiwara, M. Jimbo, T. Miwa, Transformation groups for soliton equations, in: M. Jimbo, T. Miwa (Eds.), Proceedings of RIMS symposium on Non-linear Integrable Systems-Classical and Quantum Theory, World Scientific, Singapore, 1983, pp. 39-119.

[6] L.A. Dickey, Soliton equations and Hamiltonian Systems, 2nd ed., World Scintific, Singapore, 2003, p. 420.

[7] M.H. Li, J.P. Cheng, C.Z. Li, J.S. He, The recursion operators of the BKP hierarchy and the CKP hierarchy, Sci. Sin. Math. 43 (2012), 499-514 (in Chinese).

[8] Y. Nambu, Generalized Hamiltonian dynamics, Phys. Rev. D. 7 (1973), 2405-2412.

[9] G. Papadopoulos, M2-branes, 3-Lie algebras and Plücker relations, J. High Energ. Phys. 05 (2008), 054.

[10] L. Takhtajan, On foundation of the generalized Nambu mechanics, Comm. Math. Phys. 160 (1994), 295-315.

[11] X.L. Wang, J.Q. Mei, M.L. Li, Z.W. Yan, On generalized Lax equations of the Lax triple of the BKP and CKP hierarchies, J. Nonlinear Math. Phys. 24 (2017), 171-182.

[12] X.L. Wang, L. Yu, Y.X. Yang, M.R. Chen, On generalized Lax equation of the Lax triple of KP hierarchy, J. Nonlinear Math. Phys 22 (2015), $194-203$.

[13] H.X. Wu, X.J. Liu, Y.B. Zeng, Two new multi-component BKP hierarchies, Commun. Theor. Phys. 51 (2009), 193-199.

[14] Y.X. Yang, S.K. Yao, C.H. Zhang, W.Z. Zhao, Realization of the infinite-dimensional 3-algebras in the Calogero-Moser model, Chin. Phys. Lett. 32 (2015), 040202. 